\title{
Ocular manifestations during sarcoidosis: about 13 cases. Experience of the Military Hospital Mohamed V of Rabat
}

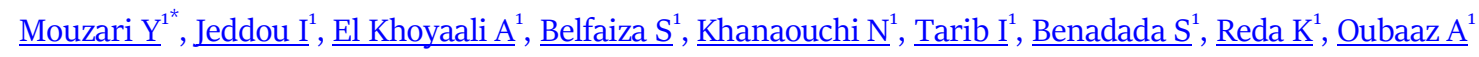 \\ ${ }^{1}$ Department of Ophthalmology, Military Hospital, Mohammed V University, Morocco
}

Corresponding Author: Y Mouzari

Address: Department of Ophthalmology, Military Hospital, Mohammed V University, Morocco.

Received date: 20 March 2019; Accepted date: 23 April 2019; Published date: 29 April 2019

Citation: Mouzari Y, Jeddou I, El Khoyaali A, et al., "Ocular manifestations during sarcoidosis: about 13 cases. Experience of the Military Hospital Mohamed V of Rabat”. Asp Biomed Clin Case Rep, vol.2, no.1: 13-17, 2019.

Copyright (C) 2019 Mouzari Y, Jeddou I, El Khoyaali A, et al., This is an open access article distributed under the Creative Commons Attribution License, which permits unrestricted use, distribution, and reproduction in any medium, provided the original work is properly cited.

\section{Keywords}

Ocular Manifestations; Sarcoidosis; Dyspnoea; Hypercalcaemia

\section{Introduction}

Sarcoidosis, or Besnier-Boeck-Schaumann disease, is systemic granulomatosis of an unknown etiology characterized by the formation of immune granulomas in the affected organs. The clinical presentation can be very variable [1]. The diagnosis is made on a bundle of clinical, paraclinical and anatomopathological arguments when a biopsy is performed. The most commonly affected organs are the mediastinal lymphatic system, lungs, skin, and eyes.

Ocular manifestations of sarcoidosis could take very different forms. We could find it in one in four patients and it may be revealing of sarcoidosis in 19\% of cases [1]. All the tunics of the eyeball and the ocular adnexa can be involved.

\section{Patients and Methods}

We report the results of a retrospective study conducted in the ophthalmology department of the Military Hospital Mohamed V of Rabat during a period of 3 years.

We have included the patients treated in the ophthalmology department of the Military Hospital Mohamed V of Rabat between January 2016 and January 2019. The diagnosis of sarcoidosis was retained on the presence of a bundle of clinical and paraclinical proofs, including at least anatomopathological evidence and/or a hyperlymphocytosis with a CD4/CD8 ratio exceeded 5 in the bronchoalveolar lavage. Furthermore, the diagnosis of ocular sarcoidosis was based on a compatible ophthalmic presentation and histological proof of granulomatosis.

We have collected the clinical, paraclinical, therapeutic and evolutionary characteristics of the patients including:

I. Clinically: the age and the sex of the patient, functional signs, visual acuity, biomicroscopy examination of the anterior segment and the fundus, eye tone measured with Goldman tonometer, gonioscopy and the results of fluorescein angiography and optical coherence tomography.

II. Paraclinically: the dosage of the angiotensinconverting enzyme (ACE) and corrected calcemia, chest radiograph, thoracic computed tomography scan, cardiac ultrasonography, brain magnetic 
Citation: Mouzari Y, Jeddou I, El Khoyaali A, et al., "Ocular manifestations during sarcoidosis: about 13 cases. Experience of the Military Hospital Mohamed V of Rabat”. Asp Biomed Clin Case Rep, vol.2, no.1: 13-17, 2019.

resonance imaging, tuberculin intradermal reaction, electrocardiogram, bronchoalveolar lavage (to precise the percentage of lymphocytes and the $\mathrm{CD} 4 / \mathrm{CD} 8$ ratio), the location and the number of biopsy.

III. The therapies are given to the patients and their dosage: coticotherapy, biotherapy (Infliximab), synthetic antimalarials (hydroxychloroquine), topical and systemic glaucoma medication and the possible recourse of cataract surgery, vitrectomy or trabeculectomy.

IV. Evolutionarily: the overall duration of follow-up, duration of oral corticosteroid therapy, the free interval before recurrence at discontinuation of corticosteroids, degree of recovery, the appearance of macular edema during follow-up and/or the persistence of refractory macular edema.

In total, 13 patients were included in the study with a follow-up of 25 eyeballs (one patient had unilateral ocular involvement).

\section{Results}

The average age at the diagnosis was 51 years with extremes ranging from 19 to 79 years. The majority were women $(77 \%)$. The median free interval between the diagnosis of sarcoidosis and the first ophthalmologic sign was six years [2-10]. The median follow-up of the patients was 36 months (between 7 and 130 months). Nine patients (69\%) had initially a systemic involvement associated with the ocular manifestations of the sarcoidosis, while the disease was strictly ocular at the time of diagnosis in

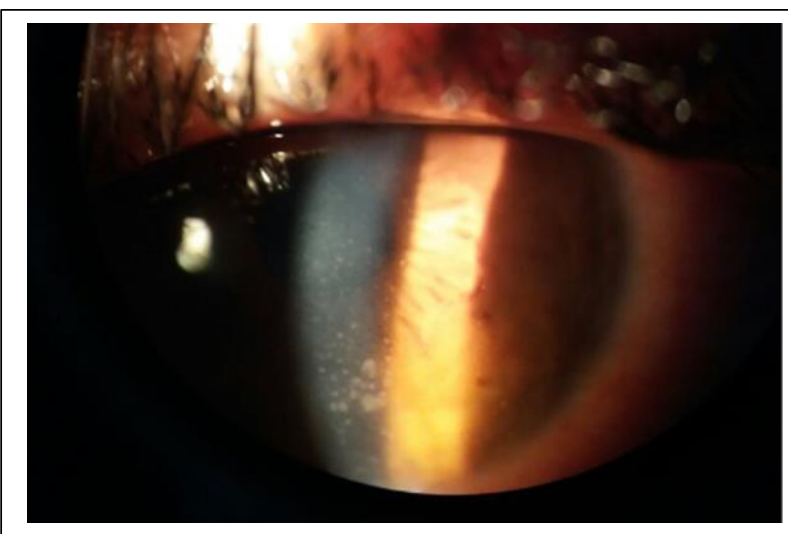

Fig-1: Granulomatous mutton-fat keratic precipitates five cases.

The main ocular functional signs were: visual acuity decline in $84 \%$ of the cases and eye pain in $53 \%$ of the cases. Meanwhile, the systemic signs were as follows: arthralgia in $38 \%$ of the cases, myalgia in $23 \%$, dyspnoea in $15 \%$ of the cases and nervous system involvement in $15 \%$ of the patients (Table-1).

\begin{tabular}{|l|l|l|}
\hline \multicolumn{2}{|c|}{ Table-1: Clinical characteristics of the patients } \\
\hline \multicolumn{2}{|l|}{ Number of patients } & 13 \\
\hline \multirow{2}{*}{ Sex } & Women & $77 \%$ \\
\cline { 2 - 3 } & Men & $23 \%$ \\
\hline Average age at the diagnosis (years) & 51 \\
\hline Median follow-up (months) & $36(7-130)$ \\
\hline $\begin{array}{l}\text { Median free interval between the } \\
\text { diagnosis and the first ophthalmologic } \\
\text { sign (years) }\end{array}$ & $6(2-10)$ \\
\hline
\end{tabular}

The ophthalmological involvement was bilateral in $92 \%$ of the cases. One patient presented hypertrophy of the lacrimal gland with ptosis. The slit lamp examination found $69 \%$ of anterior uveitis with $56 \%$ of Keratic precipitates (Fig-1), 38\% of posterior synechiae between the iris and the capsule of the lens (Fig-2), iris nodules in 15\% (Koeppe in all the cases).

The eye tone measured with Goldman tonometer showed initially a median of $17 \mathrm{mmHg}$ (with extremes betwen12 and $50 \mathrm{mmHg}$ ). Ocular hypertonia over than $25 \mathrm{mmHg}$ was found in 30\% of the cases. 
Citation: Mouzari Y, Jeddou I, El Khoyaali A, et al., "Ocular manifestations during sarcoidosis: about 13 cases. Experience of the Military Hospital Mohamed V of Rabat”. Asp Biomed Clin Case Rep, vol.2, no.1: 13-17, 2019.

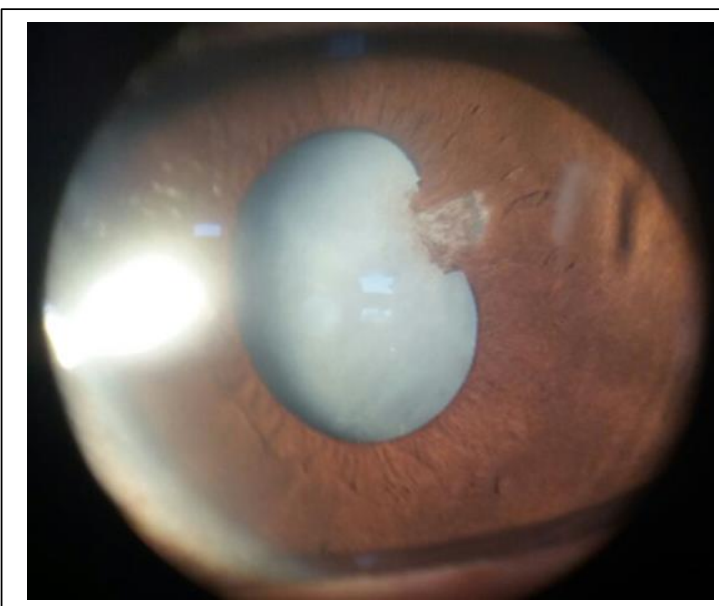

Fig-2: Posterior synechiae between the iris and the capsule of the lens

The examination of the fundus showed $30 \%$ of vitritis and $15 \%$ of papillary edema and $15 \%$ of macular edema that was confirmed by the macular optical coherence tomography. Otherwise, the fluorescein angiography revealed $38 \%$ of retinal vasculitis that was associated with retinal ischemia in $7.6 \%$ of the cases and with multifocal choroiditis in $23 \%$ of the cases.

The biological investigation found an elevated level of the angiotensin-converting enzyme in $61 \%$ of the patients and a hypercalcaemia (albumin-corrected serum calcium) in $7.6 \%$ of the patients while the tuberculin intradermal reaction was negative for all the patients.

In lungs, chest radiography was abnormal in $46 \%$ of cases, thoracic computed tomography scan found abnormalities in $15 \%$ of the cases and the bronchoalveolar lavage found an hyperlymphocytosis with a median of 0.4 [0.2-0.7] in $61 \%$ of the patients with a positive $\mathrm{CD} 4 / \mathrm{CD} 8$ ratio in $53 \%$ of the patients.

The biopsies was made in the lungs and found an epithelioid granuloma in $85 \%$ of the patients. Regarding the therapeutic side, we prescribed eye drops containing corticosteroids in $85 \%$ of cases and hypotonizing eye drops in $30 \%$ of cases $(50 \%$ of whom received oral or intravenous acetazolamide to control high hypertonia). 30\% of the patients benefited of one or more boluses of Solumedrol and $70 \%$ of them benefited of oral corticosteroids at the dose of $1 \mathrm{mg} / \mathrm{kg}$ per day followed by a gradual decline. The median duration of oral corticosteroid therapy was 12 months.
During follow-up, $23 \%$ of the patients underwent cataract surgery and 7\% underwent trabeculectomy. As a result of the initiation of anti-inflammatory therapy, $85 \%$ of the patients have had a rapid recovery. Recovery was considered partial in 53\% of cases, and total in 30\% of cases. The recurrences concerned 4 patients.

\section{Discussion}

Sarcoidosis is a chronic systemic granulomatous disease of unknown origin that can reach all the organs (lungs, lymphatic system, skin, and eye). The ophthalmological involvement is relatively frequent and occurs in $25 \%$ of the cases [2]. It is the inaugural manifestation in $20 \%$ of the cases and may precede pulmonary manifestations by several years [3].

Almost all elements of the eyeball, ocular adnexa and orbit may be affected by this disease. Although the involvement of the anterior segment is the most common, it's the involvement of the posterior segment that endangers the visual prognosis [4]. The disease is usually bilateral.The conjunctival and lacrimal gland disorders are relatively frequent but they do not significantly affect the visual functional prognosis. In contrast, ocular complications such as retinal ischemia with neovascularization and isolated optic nerve damage are relatively rare but affect significantly the functional prognosis. The most common ocular manifestations observed during sarcoidosis are uveitis. All types of uveitis can be seen, from simple unilateral anterior uveitis to severe bilateral panuveitis. Otherwise, the uveitis presents a 
Citation: Mouzari Y, Jeddou I, El Khoyaali A, et al., "Ocular manifestations during sarcoidosis: about 13 cases. Experience of the Military Hospital Mohamed V of Rabat”. Asp Biomed Clin Case Rep, vol.2, no.1: 13-17, 2019.

difficult management problem due to its chronic evolution and its functional repercussion (Table-2) [4].

The first symptoms of ocular sarcoidosis occurred in patients over 40 years old in $61 \%$ of the cases. Classically, pulmonary sarcoidosis occurs on average before 40 years old [5]. However, the mode of occurrence of the disease is different according to the ethnic origins of the patients. Sarcoidosis is a more common in black population with à femalepredominant [6], suggesting genetic susceptibility.

Many studies have been done on the genes of the HLA system. The results are in favor of susceptibility for HLA typing A1, B8 and DR3 [7].

According to the data of the literature, the

\begin{tabular}{|l|l|}
\hline \multicolumn{2}{|c|}{ Table-2: Clinical characteristics of the patients } \\
\hline Conjunctiva & Conjunctival nodules, Cicatricial symblepharon or entropion, dry syndrome \\
\hline Sclerotic & Scleritis, episcleritis \\
\hline Cornea & Interstitial keratitis \\
\hline Uvea & Anterior, intermediate or posterior uveitis, panuveitis \\
\hline Lens & Cataract \\
\hline Retina & Retinal ischemia with neovascularization, vasculitis, macular edema \\
\hline Choroid & Choroidal granulomas, neovascularization \\
\hline Optic nerve & $\begin{array}{l}\text { Clinical papillary edema, optic atrophy, optic nerve granuloma and prepapillary } \\
\text { neovascularization }\end{array}$ \\
\hline Lacrimal gland & Keratoconjunctivitis sicca, lacrimal hypertrophy \\
\hline Eyelids & Granuloma, ptosis \\
\hline Orbit & Diffuse unilateral orbital inflammation \\
\hline
\end{tabular}

paraclinical tests performed for the diagnostic have variable degrees of sensitivity and specificity and none of them has $100 \%$ sensitivity or specificity.

The 2017 Revised criteria of the International Workshop on Ocular Sarcoidosis (IWOS) for the diagnosis of ocular sarcoidosis [8] identifies seven clinical intraocular signs and five ancillary tests suggestive of ocular sarcoidosis. In our serie, two of the seven clinical signs were found frequently: mutton fat keratic precipitates and/or iris nodules (Koeppe/Bussaca) in $56 \%$ of the cases and bilateralil inflammation in $92 \%$ of cases). Goniosynechiae (38\%) and choroidal nodules (23\%) were also present.

Four of the five ancillary tests were represented: tuberculin anergy (100\%), Elevated angiotensin- converting enzyme (61\%), bilateral hilar lymphadenopathy showed in Chest X-ray (46\%) and only found in Chest tomography (15\%). Liver-enzyme tests was normal in all the patients of the study.

\section{Conclusion}

Sarcoidosis is a multisystemic inflammatory disease. Its expression seems to result from a complex interaction between environmental factors and genetic susceptibility with many different genes [9,10]. The genes of the HLA system have been studied. The results showed a correlation between the HLA phenotype and the risk of occurrence of the disease in one side, its mode of presentation or its evolutionary profile in another side. These associations seem to vary according to geography and ethnicity $[9,10]$. The diagnostic approach requires a lot of clinical and paraclinical investigations [11], involving a 
Citation: Mouzari Y, Jeddou I, El Khoyaali A, et al., "Ocular manifestations during sarcoidosis: about 13 cases. Experience of the Military Hospital Mohamed V of Rabat”. Asp Biomed Clin Case Rep, vol.2, no.1: 13-17, 2019.

collaboration between internists, ophthalmologists and pulmonologists.

If we suspect ocular involvment of sarcoidosis, the first line of tests should be: tuberculin intradermal reaction, angiotensin-converting enzyme, chest CT scan, brain MRI and biopsy of the accessories salivary glands.

The tests that could be discussed in a second-line, if the clinical examination is highly suggestive is the bronchoalveolar lavage (to precise the percentage of lymphocytes and the $\mathrm{CD} 4 / \mathrm{CD} 8$ ratio). In our study, lungs biopsies that had been performed during the bronchoalveolar lavage had found histological evidence in 11 out of 13 cases ( $85 \%$ ).

\section{References}

[1] Hariz H, Marzouk S, Ben Salah R, et al, "Les manifestations oculaires au cours de la sarcoïdose”. La Revue de Médecine Interne, vol.33, suppl.2 : A168, 2012.

[2] COMHAIRE-POUTCHINIAN Y, "UVÉITE SARCOIDOSIQUE. Bull. Soc". belge Ophtalmol, vol.277: 57-63, 2000.

[3] Obenauf CD, Shaw HE, Sydnor CF, et al., "Sarcoidosis and its ophthalmic manifestations". Am J Ophthalmol, vol.86, no.5: 648-55, 1978.
[4] Baughman RP, Lower EE, Kaufman AH, "Ocular sarcoidosis”. Semin Respir Crit Care Med, vol.31, no.4: 452-62, 2010.

[5] Lemos-Silva V, Araújo PB, Lopes C, et al., "Epidemiological characteristics of sarcoidosis patients in the city of Rio de Janeiro, Brazil”. J Bras Pneumol, vol.37, no.4: 438-45, 2011.

[6] Swigris JJ, Olson AL, Huie TJ, et al., "Sarcoidosisrelated mortality in the United States from 1988 to 2007”. Am J Respir Crit Care Med, vol.183, no.11: 1524-30, 2011.

[7] Martinetti M, Tinelli C, Kolek V, et al., ““The sarcoidosis map": a joint survey of clinical and immunogenetic findings in two European countries". Am J Respir Crit Care Med, vol.152, no.2: 557-64, 1995. [8] Mochizuki M, Smith JR, Takase H, et al., "Revised criteria of International Workshop on Ocular Sarcoidosis (IWOS) for the diagnosis of ocular sarcoidosis”. Br J Ophthalmol: 313356, 2018.

[9] Iannuzzi MC, Rybicki BA, "Genetics of sarcoidosis: candidate genes and genome scans". Proc Am Thorac Soc, vol.4, no.1: 108-16, 2007.

[10] Smith G, Brownell I, Sanchez M, et al., "Advances in the genetics of sarcoidosis". Clin Genet, vol.73, no.5: 401-12, 2008.

[11] Bodaghi B, Touitou V, Fardeau C, et al., "Ocular sarcoidosis”. Presse Med, vol.41: 349-54, 2012. 